\title{
AN OVERVIEW OF WIRELESS BASED SUBSEA TUNNEL MONITORING SYSTEM
}

\author{
Gopinath Marefeen ${ }^{1}$, Gowrishankar Kasilingam², Mritha Ramalingam³ \\ ${ }^{I}$ Faculty of Engineering \& Computer Technology, AIMST University, Kedah, Malaysia \\ ${ }^{2}$ Faculty of Engineering \& Computer Technology, AIMST University, Kedah, Malaysia \\ ${ }^{3}$ School of Engineering, Universiti Sains Malaysia, Nibong Tibal, Malaysia
}

\begin{abstract}
In this paper an overview of subsea tunnel monitoring systems which exist in the related field of research are discussed. A reliable monitoring system with a remote access to monitoring parameter data for subsea tunnels using wireless technology is very crucial as it offers huge advantages as compared to the other existing monitoring systems. The subsea tunnels are often subjected to considerable sources of threats due to the complex geological conditions, unpredictable events of natural disaster and complex structural integrity risk involved. In order to ensure the safety of the subsea tunnel structures, it is a vital necessity to continuously monitor the structural health parameters of the tunnel 24/7 a year, as compared to usual visual inspection conducted by dedicated team of civil and structural engineers in a periodic term. This paper also presents proposed wireless monitoring system and a brief experimental setup of the whole system. Here the proposed system combines the characteristics of subsea tunnel monitoring system and several other research works relating to the field of study to put forward a general survey on subsea tunnel monitoring system through wireless application. This paper is a comprehensive overview of the literature and does not contain implementation results.
\end{abstract}

Keywords: Subsea tunnel, Monitoring system, Wireless.

\section{INTRODUCTION}

When Thames Tunnel was successfully engineered and constructed in 1843, which is often described as the Eighth Wonder of the World, it set history as the first underwater tunnel, and structural architecture design of this kind in the world. This has marked the evolution of subsea tunnel as the next form of engineering solution for structural development involving land connectivity.

Before moving on further, let us explain what a subsea tunnel is? A subsea tunnel is a passageway completely enclosed except for openings for entrance and exit in which it is structurally erected beneath the surface of the sea. It is one of the most complexly engineered structures in the world due to the huge risk involved. Any untoward structural collapse may claim thousands of lives in a glimpse. Subsea tunnel becomes the only option in some isolated cases where operating a ferry link or constructing a bridge is impossible.

We may wonder why engineers would want to uptake such an expensive, complex and riskiest project like the subsea tunnel engineering, while they may easily opt for bridge as the easiest solution. Well, the reasons are as so. A staggering 90 percentage of the world's commodity and merchandise trade are transported by ships, thus

ports are very important to the wellbeing and health of the global economy. Meanwhile this percentage has remained fairly the same in the last century, volumes of this trade have increased rapidly in the past two decade, and this number is expected to be growing exponentially in the future. A sea port is a location on a coast or shore of a country that contains multiple harbors to allow ships to dock and transfer people or cargo. A good economic growth of any country is reflected back to its sea port performance. Citing Singapore as a perfect example, currently it is the world's secondbusiest port in terms of total shipping tonnage. Singaporean port transships a fifth of the world's shipping containers, half of the world's annual supply of crude oil, and is the world's busiest trans-shipment port. Many countries have marveled at how this small island, smaller than a New York City, has come to be worth more than around $\$ 300$ billion US dollar. One key aspect of Singapore remarkable economic success despite its size is the vibrant shipping port.

However, building a bridge would adversely affect the whole sea traffic to a particular port of a country due to certain constrains. If a bridge is to be constructed, it would need a wide opening or swing bridge to allow shipping to pass. Even if the opening on the bridge is wide enough for ships to pass through, the ship traffic movement will be riskier, leading to serious accidents which may occur. Due to these inconveniences, increasing number of ships may shy away from visiting such sea ports. This indirectly affects the port business and reflects on to the economy of the particular country. If a subsea tunnel was to be constructed, it would overthrow all the downfalls of a sea bridge, thus allowing free flow of shipping activities and sea traffic without any structural obstacle. 
There are also other minor downfalls of bridge constructions as compared to a subsea tunnel. The other advantage gained by engaging in subsea tunnel engineering is space. New land space at prime urban seafront areas can be created through underwater tunneling with the soil that has been excavated, as in the case of Penang island of Malaysia, where rapid land reclamations is being done for land development in the state.

One of the notable examples of existing subsea tunnel in the world is Seikan Tunnel, which runs between the islands of Hoshu and Hokkaido in Japan. It is the world's longest undersea railway tunnel with a distance of $53.9 \mathrm{~km}$ long, together with non-undersea portions of the tunnel are also accounted for. Next is the Channel Tunnel, which is a rail link between England and France. It is the world's longest undersea portion railway tunnel, which measures $37.9 \mathrm{~km}$ long. Eiksund Tunnel, a new subsea tunnel, which set new world record for deepest under-sea tunnel in Norway, with a depth of about 266 meters under the sea level. Last but not least, Russian Bering Sea tunnel would be soon the world's longest, which is currently under planning stage. It is proposed to run under the sea of the Bering Strait and connect Russia directly to Alaska. The total length of the subsea tunnel is about $6,000 \mathrm{~km}$ of infrastructure, with thirteen years of estimated completion time. On the next section, a literature survey study on various combination of past publication journals, articles and online materials were made and reviewed.

\section{LITERATURE REVIEW}

Subsea tunnel monitoring system is a growing field of research that is attracting increasing interest from government agencies as well as many other engineering societies in order to maintain the safety of the overall subsea tunnel. For a particular subsea tunnel monitoring system, there are different monitoring parameters, approaches and technologies involved. A literature survey study on various combination of past publication journals, articles and online materials were made to further dwell into the field of project proposed.

According to publication by FORCE Technology, they presented a unique system for monitoring of reinforcement corrosion in concrete structures in conjunction with the construction of China's first subsea tunnel [5]. It was understood that this system was proposed to optimize the maintenance strategy and also to ensure a long service life, by installing a corrosion monitoring system in the tunnel. Department of Materials Science and Engineering Fujian Key Laboratory of Advance Materials Xiamen University, proposed the study on Applications of embedded sensors for in-situ corrosion monitoring in subsea tunnel steel corrosion in reinforced concrete structures, particularly under coastal marine environments, which is the main factor that influences the durability of concrete structures. In order to monitor corrosion of steels in reinforced concrete structures of subsea tunnel of China, the commercial CorroWatch multi-sensors and the self-made ladder sensors were embedded in the selected critical concrete structures during the constructions of subsea tunnel. Correlations among the measured parameters and corrosion situation are discussed based on the real-time measured data. Corrosion monitoring thus supplies an early warning of reinforcement corrosion.

On the study of developing wireless vibration sensor for tunnel construction and maintenance [15], the advantages and usefulness of the wireless automatic monitoring system is addressed compared with the conventional wired or survey measurement systems. A framework of the process for monitoring, manipulating, and transferring data from MEMS sensor was studied and the applications of the newly developed automatic monitoring system for tunnel monitoring were mentioned. The wireless-based monitoring system is based on small wireless vibration sensor that was designed and developed. It is addressed that wire-based systems can only be applied for a limited area and the systems become incapable if the wire is cut, which happens frequently. In order to overcome those disadvantages and limitations of monitoring systems, the wireless-based monitoring systems, which are based on small wireless vibration sensors, is used for tunnel monitoring purpose.

As per the next review, for a tunnel monitoring system type used, it was presented about the development and the results of a fast method for displacement measurement based on digital images [1]. This technique allows a deformation analysis along the cross-sections of a tunnel. It was also understood that this technique was created to overcome some drawbacks of most traditional sensors employed, especially in the case where some sensor technology limits the number of cross-sections that it is capable to be checked under a specified circumstances. Besides, based on the journal survey, they've admitted that only a combination of different instruments and sensors can provide sufficient information to monitor the deformations to predict the behavior of the structures of a tunnel. In the case of complex structures like subsea tunnel, only a monitoring system based on combined sensors allows a complete and detailed analysis of the object and its surroundings, with different accuracies according to the requests of civil engineers. Another discussion about the deformation monitoring via automatic sensors, which are permanently fixed on the structure, is also done. It is stressed that the choice of design for automated monitoring via sensors depends on many factors, for example, the period of the monitoring, the level of risk for people or infrastructures potentially involved the shape of the object, the measurement accuracy, the installation costs, the number of investigated points and their distribution and so on.

Moving on further, through the analysis of hydrodynamic pressure on circular subsea tunnel in saturated soil, various summaries were made [4]. Starting from the theory of soil, water and solid structure dynamic interaction, the paper investigated the analysis model and method of the coupled system. Through the computation and comparison of hydrodynamic pressure of four calculation cases of circular subsea tunnel by ANSIS software, it is found that the compressibility of water and the flexibility of tunnel lining 
play an important role in the vibration analysis of the integrated system. The finite element method has been utilized to calculate the hydrodynamic pressure of a long, submerged, circular subsea tunnel subjected to horizontal ground excitation. The problem was idealized as twodimensional, and ANSYS software was used to solve the coupled fluid-structure interaction problem in the analysis. Through the numerical analysis, it is found that the hydrodynamic pressure on the tunnel surface is mainly influenced by the compressibility of seawater and the flexibility of lining. The larger the rigidity for the structure, the smaller the pressure that we can get, furthermore, compared with compressible water, the hydrodynamic pressure on lining surface is downward in uncompressible seawater.

Tunnels are challenging spaces in terms of safety [2]. The author stressed that, compared to an open space, evacuation possibilities for a subsea tunnel is very limited and rescue personnel needs additional equipment to reach underground locations in the case of an emergency. Safety issues in operational phase are evident factors that influence the possibility and feasibility of a tunnel project. The main challenges regarding safety in tunneling are evidently the different kinds of accidents involved. In a subsea tunnel, the most severe risk scenarios are fire and flooding. Other important factors are reliable operation and handling of aberrant situations. Prevention of potential risks in advance and a rapid response are the key guidelines when planning a safe long subsea tunnel. It is vital to make detailed plans of action to be prepared for emergency situations. It was made clear that a good understanding on the surrounding environment is important in order to select the best possible solutions as basis for tunnel planning.

In another point, the author highlighted in the article that the sea level is not constant, but fluctuates continuously according to prevailing weather conditions. Special weather conditions such as storms and bath effect may raise sea level temporarily by another meter. On top of this, wave's causes rise of effective water level too. As water inflow to a subsea tunnel would be catastrophic, conservative safety margins should be applied. It is also warned that the most notable safety risk in subsea tunnel is a sudden water inflow accident. Keys to avoid problems during tunnel operation are careful monitoring of deformations in constructed tunnel.

The safety and self-rescue gets more complicated as a subsea tunnel's length increases [3]. In the case of an emergency or unfavorable condition in the tunnel, the usual practice is to be informed to rescue personnel by road users through emergency hotlines. By the time rescue personnel arrive to the scene, situations could have gone worse and rescue operation would be cumbersome. Furthermore, while the subsea tunnel increases in length, the time needed for the usual safety response will be longer as well. Thus, an automated monitoring and response reaction towards potential problem is crucial in this case. The author proposed about a ventilation system which was specially designed for a subsea tunnel structures. The system comprises of normally self-ventilated tubes by means of the piston effect caused by traffic supported by longitudinal ventilation by jet fans from portal to portal. The ventilation system is applied for both the day to day operation, as well as for emergency situations, like in the event of fire. The ventilation design for the tunnel takes into account the varying traffic volume throughout the day, the expected traffic growth in the future and reduced car emissions through technological improvements. The system is capable of keeping conditions in the tunnel below internationally recognized threshold values throughout the whole lifetime of the tunnel.

There are two types of tunnel ventilation systems, longitudinal and transverse ventilation [13]. In the event of tunnel fire, smoke propagation along the tunnel is one of the key features of fire ventilation design especially in subsea tunnels. In a transversely ventilated subsea tunnel, the smoke propagating distance increases with the fire size and decreases with the exhaust ventilation rate. The supply rate does not affect the smoke propagating distance when it is smaller than the exhaust rate. If the supply rate is larger than the exhaust rate, the whole cross-section of the tunnel will be filled with smoke, which is clearly a negative phenomenon in the fire safety design. The smoke propagating distance and the smoke descent with ventilation rates of supply and exhaust may serve as good criteria in the fire safety of transversely ventilated subsea tunnels.

The use of a longitudinal ventilation system for smoke control is based on the assumption that it will be under normal operation and absence of road traffic congestion in the subsea tunnel. In the event of a fire in the subsea road tunnel, the traffic in front of the fire should be informed immediately to continue to drive through the tunnel towards the exit point, travelling faster than the flowing smoke layer.

Relating to the publication "The whole life of subsea tunnel safety monitoring and measurement technology" [7], a number of scientific research achievements by Xiamen Xiangan subsea tunnel were discussed. The author discussed about various tunnel risk identification method, tunnel safety monitoring level of risk based on the standard of grading, tunnel-life safety monitoring and rating method, subsea tunnel life safety monitoring risk factors identification and so on. The relationship between subsea tunnel variation in morphology and monitoring level were also clearly analyzed. Another important factor, which is the threedimensional displacement measurement method, displacement information acquisition device, stress and earthquake and the durability of the monitoring technology of subsea tunnel structure were detailed.

On the study of 'Durability Monitoring of Concrete Structure of Subsea Tunnel' [6], the authors mentioned that the subsea tunnel at Jiaozhou bay is the second self-built tunnel in China with the designed service life for over 100 years. The anode-ladder-system is used to monitor the chloride ion penetration into reinforced concrete structures 
in a corrosive groundwater and saline-fog environment. An expansion-ring-system is used to monitor the water absorption level by concrete linings, and the durability of concrete structure of subsea tunnel is assessed according to the monitoring data obtained. The anode-ladder-system utilizes monitoring sensors which are embedded into the subsea structure's concrete, the external concrete linings facing the rock zones of sea portion, and at the entrance of the subsea tunnel. The measurements from sensors installed at the concrete structure were collected serially for 1 year and its trends were observed. According to the monitoring results obtained, the durability of concrete structure for subsea environment is deemed good.

As to monitoring the structural integrity of a subsea tunnel structure, a method for calculating convergences in tunnels by means of measuring strains at several points of its contour is described [8]. Deformations with respect to time can be measured either by using electrical resistance strain gages or fiber optics Bragg gratings attached to the steel arches of tunnel support, or bars that may be installed along vertical sections of the tunnel. These strains are continuously converted into convergences so that it would trigger alarm signals whenever tunnel stability is under risk, and would be instantaneously transmitted to a remote computer network in order to be closely surveyed by management authorities. Some illustrative examples on the said method were presented for both laboratory and field applications by this author to support his claim on its reliability.

In the publication of "Development of an IT-based Monitoring System for Mountain Tunnel", a systematic monitoring approach was established in an environment where the monitoring information of mountain tunnel construction is disseminated and handled through Internet [9]. This implementation allows on-site staff and off-site stakeholders to possess a credible management, visualization and analysis tools of monitoring data. Acquisition of monitoring data, data processing and analysis is fast and efficient, and the monitoring results can be relayed to the engineers on time. An alarming system consisted of five alarming levels is also proposed to ensure the safety of tunnel construction. Empirical alarming values of subsidence of tunnel crown and convergence deformation of tunnel outline are derived based on actual observation values of the particular investigated project. It is also to be noted that the whole proposed system is capable of managing multiple tunnels at the same time, right from the customized monitoring sections and monitoring parameters, and $3 \mathrm{D}$ visualization of the monitoring results.

Through the survey on "Basic studies on earthquake damage to shallow mountain tunnels", it is understood that as tunnels are surrounded by ground, they are highly resistant to earthquakes as long as the ground is stable [10]. Tunnels located in stable ground do not require a seismic design. However, tunnels are often susceptible to tremors depending on the scale of the earthquake and the distance from the epicenter. A several case studies of earthquake damage, the behavior of an actual tunnel measured in earthquake conditions and the results of a prototype model test implementation to clarify the mechanism of earthquake damage were investigated by the author.

A review on damage case histories and protection methods under seismic loading [11], revealed several case history collection of damaged tunnel, damage classification criterion, seismic parameters affecting the tunnel damage, types of cracks along the tunnel lining and the available protection methods. Soil condition parameter is identified as one of the factor leading to structural cracks to a tunnel. Summary of crack types were also explained in detail, such as sheared off lining, slopes failure induced tunnel collapse, longitudinal cracks, transverse cracks, inclined cracks, extended cracks, and wall deformation.

As in the publication "Wireless underground sensor networks", a Wireless Underground Sensor Network or abbreviated as WUSN, was used to demonstrate the application of wireless technology as monitoring solutions via the sensor networks, [12]. A variety of different parameter conditions and monitoring work can be executed using WUSN. WUSN devices can be deployed completely below sea level with no wired connections. It was understood that establishing a good wireless communication within a dense substance such as soil, seabed, seawater and rock is, however, significantly more challenging than through air. This factor, combined with the necessity to conserve energy due to the difficulty of unearthing and recharging WUSN devices, requires the particular communication protocols to be designed to be as efficient as possible. WUSNs are able to wirelessly forward sensor readings to a central sink in real time and the devices could be embedded within key structural components of a subsea tunnel to monitor stress, strain, leakage, damage and other parameters.

It is also identified that the five main factors, however, which impact communication with Electromagnetic (EM) waves in the underground level is the extreme path loss, reflection and refraction, multi-path fading, reduced propagation velocity, and noise. Besides, it is stated that low frequencies are capable of propagating with lower losses through the underground environment, and that frequencies used by traditional terrestrial Wireless Sensor Network (WSN) are infeasible for this type of underground environment. The use of low frequencies, however, severely restricts the bandwidth available for data transmission in WUSNs.

In a subsea tunnel, the composition of a seabed, including its water content, particle sizes, density, and temperature, all combines to form a complex dielectric constant, $\in_{\mathrm{r}}$. This parameter directly affects the attenuation of any EM signal passing through the soil, and it is thus useful to make further investigative research on wireless data transmission in subsea tunnel. Soil water content is by far the most significant parameter to consider when predicting signal loss through a given type of soil. The channel will face 
significant losses if there is high presence of water content in the surrounding soil of the subsea structure.

A method for analysis of underwater tunnels in axisymmetric plain strain conditions was presented [14] in publication "An Elasto-Plastic Model for Analysis of Underwater Tunnels in a Strain-Softening Hoek-Brown Rock Mass". The newly developed technique proposes a finite difference numerical method for calculating distribution of pore water pressure as a consideration for stress and strain around a circular tunnel. The behavior of the rock mass surrounding the tunnel is considered as elastoplastic in a strain-softening model. The model takes into account the effects of increment in elastic strain within the plastic zone as well as the di-latency angle. A more accurate model for calculating distribution of pore pressure in the elastic zone is determined by modifying the existing tested models. It is identified that small changes in height of the groundwater level does not significantly affect the evaluations for the elasto-plastic radius and ground deformation. As a result of the high initial stress of the tunnel and good quality rock mass, limited variations in groundwater level is found to have a considerable effect on the behavior of the surrounding rock mass.

As in the study of reliability of subsea tunnel in the long run, a study on the published work "The degree of deterioration of the tunnels of the Prague metro based on a monitoring assessment" pointed that every ageing of structures is a very important issue from the point of view of assessing the risk inherent in those structures [16]. A research experiment on installation and implementation of a long-term monitoring via wireless system for data collection and transfer was done. Stress change due to unloading can initiate the opening of micro-cracks for any tunnel structures. It is mentioned that monitoring solution is not directed at determining the potential risk of collapse, rather it is to confirm that the tunnel is still in a stable state and to assist relevant authorities to determine the appropriate moment to schedule repairs in order to significantly extend the expected lifetime of an underground structure. While mentioning underground structures, there is always the problem of accessibility, which leads to the use of remote monitoring based on wireless technology.

Another most common method of evaluating stability conditions of a subsea tunnel is by convergence measurements conducted by means of diametrical or perimetral changes registered by mechanical or optical systems. The extensometric method incorporated provides an excellent agreement with conventional techniques of convergence monitoring of a tunnel structure [17]. The advantages offered by the extensometric method is that greater versatility in monitoring tunnel convergences continuously, increased availability during both construction and service phases, less interference with other tunneling operations, the possibility of supplying alarm signals either sonic of red lights when excessive values are recorded, the use of wireless data transmission to display results in a network of computers, and low implementation costs.

\section{PROBLEM IDENTIFICATION}

Based on the literature study discussed in previous section, several key problems were identified. The first key problem is corrosion of metal parts in concrete structures of the subsea tunnel. One of the factors that cause corrosion on the structural metal parts is that the presence of high levels of moisture as a result of seawater interaction between the subsea tunnel's external structure and sea. As per on the tunnel monitoring system method through displacement measurement based on digital images, only a monitoring system based on combined sensor networks in a subsea tunnel would allow a complete and detailed analysis of the tunnel and its monitoring parameters, with different accuracies according to the requests of civil engineers. Moving further, through the analysis of hydrodynamic pressure on circular subsea tunnel in saturated soil, it is found that the compressibility of water and the flexibility of subsea tunnel lining play an important role in the vibration analysis of the integrated system. Excessive vibration to a subsea tunnel may cause adverse effects to the integrity of a subsea tunnels built rigidity and safety, which may lead to cracks.

Besides, subsea tunnel is a closed environment, resembling a cylindrical tube, with only an entrance and an exit at each ends. It was mentioned earlier that comparing to an open space design, evacuation possibilities for a subsea tunnel is very limited. Safety issues in operational phase of a subsea tunnel are evident factors that influence the feasibility of a subsea tunnel project. The main challenges regarding safety in subsea tunnel are evidently different kinds of in the case of occurrence of traffic accidents. In a subsea tunnel the most severe risk scenarios are fire, flooding, and sharp spike on carbon monoxide level during peak traffic. It was also cited that the most notable safety risk in a subsea tunnel is a sudden gush of water inflow in the event of a crack formation in tunnel.

An automated emergency response to trigger alarm signals whenever tunnel stability is under risk, and may be, at the same time, immediately transmit to a remote monitoring control center in order to be closely surveyed by management authorities, is another important solution that should be employed in a subsea tunnel project.

Tunnels are often susceptible to tremors depending on the scale of the earthquake and the distance from the epicenter. A proper structural health checking mechanism or a realtime monitoring system is needed to be implemented. In the event of an earthquake, an immediate access to the tunnel health parameters are needed so as to warn subsea tunnel users of the dangers ahead, rather than manual inspection that would prolong time scale and increases the risk to thousands of road tunnel users. An automated computerized response is also very crucial in order to evacuate and warn the tunnel users of the ongoing danger.

A wired monitoring system for a subsea tunnel monitoring possesses a lot of disadvantages. Wired system often vulnerable to physical damage due to unavoidable 
circumstances and events, thus it may interrupt the whole monitoring process. Embedding a wired system is also too tedious and complex, in the case of a subsea tunnel project which may extend to the range of hundreds of kilometers. Periodic maintenance is also necessary, and both installation and maintenance may cost a boom. In the case of wireless implementation of WUSNs, reliability plays an important role. A data logger may have tens of sensors connected to it and represents a single point of failure for all of them. Since the sensors of a single data logger may be spread over a large physical area, a failure of a data logger could be catastrophic to a sensing application. WUSNs however, give each sensor the ability to independently forward readings, eliminating the need for a data logger as well as the wire that must be buried between a data logger and a sensor. On top of this, WUSNs are self-healing. Device failures can be automatically routed around, and the network operator can be alerted to device failure in real time.

\section{PROPOSED SYSTEM}

\subsection{Experimental Setup}

A prototype of the subsea tunnel model with all the available design will be made and constructed. The construction of the whole layout of the subsea tunnel prototype will take into account the parameters to be monitored through the system. The subsea tunnel blocks consist of three types that are to be constructed. The first part would be the subsea tunnel structure which is embedded onto the soil zones below the seabed. The second part consist of a straight through path subsea tunnel structure which is in direct contact with sea water, and the third part being the bend part of the tunnel construction. A main microcontroller unit will serve as the central brain of the whole system, which is used to process and analyze the data obtained from the integrated multiparameter sensor network throughout the subsea tunnel blocks. All the individual sensor nodes of a network comprising integrated multi-parameter sensors would also possess its own microcontrollers each, to process the acquired data through the sensors, to be sent to the centralized unit. All the monitoring data obtained would then be linked to the centralized monitoring and control center. Wireless application would be used for the communication network throughout the subsea tunnel's monitoring system. A display screen would be constructed at the entrance of the subsea tunnel. This would act as the warning message conveyer to the subsea tunnel users as an instant response from the control center, in the event of an emergency.

Alarm siren would also be triggered through the buzzer throughout the subsea tunnel to warn subsea tunnel users in the event risk detected. The control center would also be able to control the subsea tunnel ventilation system, both with a capability of automated and manual operation. The results obtained from the multi-parameter sensors embedded into the subsea tunnel prototype, can also be relayed and accessed from this control and monitoring center by another main centralized monitoring center, which may be located very far in the capital city and relevant authority personnel in remote locations to monitor the subsea tunnel. Figure 1 shows the block diagram of proposed system.

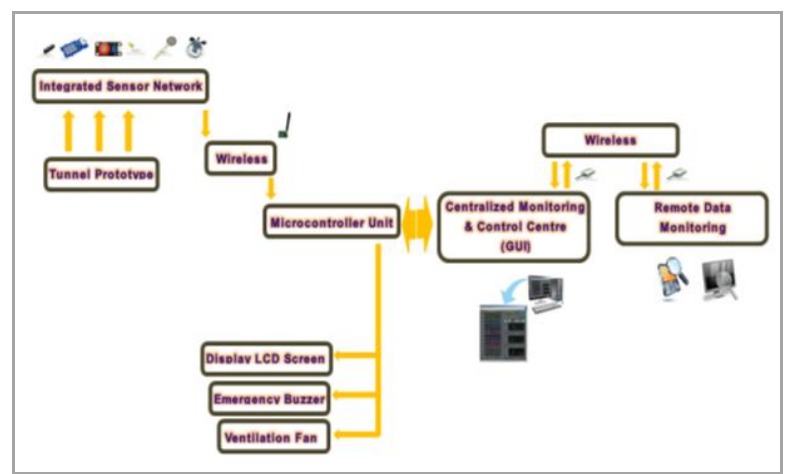

Fig-1: Block Diagram of proposed system

\section{DISCUSSION}

The objective of the whole proposed idea is on the proper reliable monitoring system for a subsea tunnel with wireless communication technology. In the event of any untoward tragedies, natural disasters and other structural safety threatening circumstances, a quicker response can be made due to the advantage of monitoring the whole subsea tunnel from a remote location in real-time. The structural integrity check of the subsea tunnel too can be accessed and monitored constantly all the time from the centralized monitoring and control center. The wireless protocol gives much more of a secure communication and ease of maintenance, than that of compared to wired connection monitoring system.

The main objective of the proposed system would be to ensure the safety of subsea tunnel and the safety of users. The earth has started reacting violently to mankind's inconsiderate and uncontrolled development throughout the past over the century which had led to global warming, leading to unusual weather conditions, including rising sea level. With uncertain natural disasters and unpredictable earthquakes occurrence, early detection of subsea tunnel condition and degradations would save thousands of lives by closing accessibility to the subsea tunnel in time. The proposed monitoring system is perfectly suited to acquire continuous and reliable monitoring data from every part of the tunnel. The system could even precisely locate the location at which the potential threat or problem is being faced in the subsea tunnel.

Extended lifetime of subsea tunnel even under ageing condition is also another plus point of proper subsea tunnel monitoring system. Some structures may visually look very dangerous for future usage and may call for demolition due to lack of confidence for the public. However, a proper continuous monitoring solution will allow the monitoring agencies of a subsea tunnel to extend its demolition if the continuous monitored data indicates safety margins are still within a healthy limit. This in turn cut cost of rebuilding a fresh new subsea tunnel, or costly repair works. In case repairs are really necessary, the monitoring data will aid engineers in designing an optimal repair framework. 
In the long run, this system monitors the subsea tunnel's structural components for indications of deterioration and fatigue, enabling the tunnel to be made more resilient and sustainable through improved maintenance. Through the implementation of subsea tunnel monitoring system, civil engineers can enhance their design considerations and parameters on future tunnel design. It greatly assists in improving and increasing knowledge on structural behavior of subsea tunnels in the long run. The continuous monitored result would serve as a database of result of the previous design work on its integrity. Any irregular patterns of results from the monitored result could be studied and further investigated for better knowledge on subsea tunnel design that would yield maximal structural integrity.

Assessing the health condition of the subsea tunnel immediately after a major event or natural disaster would be another major role of this proposed system. An early detection of the threat can be obtained in the control room of the centralized monitoring station and be notified to the relevant authorities and emergency response unit in time. The subsea tunnel can be assessed for possible damages and failures caused by extraordinary events such as earthquakes, fire, explosions or so on from the monitoring station. This ability allows quick response to this major event so that safety engineers or consultants can be deployed on time to make a preliminary assessment of whether the safety of the structure has been seriously compromised.

\section{CONCLUSION}

Subsea tunnel engineering has evolved as the next form of engineering solution for structural development involving connectivity to a greater extent, and increasing number of subsea tunnels are being constructed throughout the world, with more proposals of such project to come in the future. A proper subsea tunnel monitoring system allows rapid assessment of a subsea tunnel's state of condition. This approach is soon becoming recognized as the most reliable way to increase the safety and optimize operational, as well as maintenance works of the subsea tunnels. Data and information resulting from the monitoring system can be used to further improvise the operation, maintenance, repair and replacement of the structure based on acquired results over a period of time. Detection of on-going damages could help to discriminate deviations from the actual design performance. Structural threat to subsea tunnels can often have serious consequences and claim thousands of lives in a matter of second in case of a tragedy. The most severe are failures involving human victims. A subsea tunnel monitoring system would greatly assist on learning how a subsea tunnel performs in real conditions, and would help future engineers design better structures for the future. This can lead to more economical, durable, and much safer subsea tunnel structures with increased integrity, reliability, and safety.

\section{REFERENCES}

[1] M. Alba, L. Barazzetti, A. Giussani, F. Roncoroni, M. Scaioni (2010), "Development And Testing of A Method for Tunnel Monitoring via Vision Metrology", International Archives of Photogrammetry, Remote Sensing and Spatial Information Sciences, Vol. XXXVIII, Part 5,17-22.

[2] Timo Salmensaari (2010), "Safety in Long Subsea Rail Tunnels", Master Thesis, Helsinki, ISBN 978952-223-988-4, ISSN 1458-2198.

[3] DE WIT, J.C.W.M., Van Putten (2012), "Immersed Tunnels: Competitive tunnel technique for long (sea) crossings", Under City 2012, Dubrovnik.

[4] Chen Xianghong, Zhang Hongru, "Analysis of Hydrodynamic Pressure on Circular Subsea Tunnel in Saturated Soil", 2010, 825-829.

[5] Sacha Andersen, Brián Kofoed (2009), "Corrosion monitoring - now in China!" News Letter, Concrete News-Force Technology, No.9.

[6] Jin Zuquan, Zhao Tiejun, Zhang Peng, Gao Song (2013), "Durability Monitoring of Concrete Structure of Subsea Tunnel", Journal of the Chinese Ceramic Society, Vol.41, 205-210.

[7] Wang Mingnian (2012), "The whole life of subsea tunnel safety monitoring and measurement technology", China Communications Press.

[8] Dinis da Gama, C. (2006), "A continuous tunnel monitoring system for both construction and service stages", American Rock Mechanics Association.

[9] Xiaojun Li, Zhigang Li, Wenqi Ding, Hehua Zhu (2008), "Development of an IT-based Monitoring System for Mountain Tunnel Construction", International Association for Computer Methods and Advances in Geomechanics (IACMAG),1952-1957.

[10] H. Miyabayashi, T. Tosaka \& A. Isogai Y. Kojima \& K. Yashiro, J. Saito \& T. Asakura (2008), "Basic studies on earthquake damage to shallow mountain tunnels" Underground Facilities for Better Environment and Safety, 406-419.

[11] Giovanni Lanzano, Emilio Bilotta, Gianpiero Russo (2008), "Tunnel under seismic loading: a review of damage case histories and protection methods", Strategies for reduction of the seismic risk.

[12] Ian F. Akyildiz, Erich P. Stuntebeck (2006), "Wireless underground sensor networks: Research challenges", Adhoc Networks, Elsevier, 669-686.

[13] Byung Il Choi, Chang Bo Oh, Myung Bae Kim, Yong Shik Han, Jun Seok Choi (2006), "A New Design Criterion of Fire Ventilation for the Transversely Ventilated Tunnels", World Tunnel Congress and 32nd ITA Assembly, Seoul, Korea.

[14] Ahmad Fahimifar, Hamed Ghadami, Masoud Ahmadvand, Arvin Abdolmalek (2013), "An ElastoPlastic Model for Analysis of Underwater Tunnels in a Strain-Softening Hoek-Brown Rock Mass", International Journal of Fundamental Physical Sciences (IJFPS), Vol 3, Issue 2, 1-5.

[15] Soon-Wook Kwon, Jung-Yeo, Kim, Hyun-Seok Yoo, Moon-Young Cho, Kyong Ju Kim (2006), "Development of Wireless Vibration Sensor Using 
MEMs for Tunnel Construction and Maintenance", Vol. 21 Issue 3-4, $318-323$.

[16] Ivan Vanic `ek, Martin Vanic cek (2007), “The degree of deterioration of the tunnels of the prague metro based on a monitoring assessment", Acta Geotechnica Slovenica, 35-47.

[17] C. Dinis da Gama (2004), "A method for continuous monitoring of tunnel deformations during construction and service phases", EUROCK, Salzburg, Austria. 